\title{
Parámetros de Normalidad en Fuerzas de Prensión de Mano en Sujetos de Ambos Sexos de 7 a 17 Años de Edad
}

\author{
PAMELA ESCALONA D'A. ${ }^{1}$, JEANNETTE NARANJO O. ${ }^{1}$, \\ VERÓNICA LAGOS S. ${ }^{1}$, FRESIA SOLÍS F. ${ }^{2}$ \\ 1. Terapeuta ocupacional. Lic. en ciencias de la ocupación. \\ 2. Lic. y Magíster en bioestadística. \\ Departamento Terapia Ocupacional. Instituto de Rehabilitación Infantil Teletón.
}

\section{ABSTRACT \\ Normal Parameters in Hand Grasping Strength in subjects of both genders, 7 to 17 years of age}

Introduction: Determining normal parameters in hand grasping strength will allow to determine clinical conditions and treatment results in patients with changes in hand dexterity. Purpose: Establish ranges of normal strength in three types of grasp in subjects of both genders, ages 7 to 17. Materials and Methods: 753 random subjects selected from schools in the Metropolitan Region, two-stage sampling, $2.12 \%$ error. The following tests were performed: cylindrical palmar (dynamometer), spherical palmar (vigorimeter), and subterminolateral clasp prehension (clasp). Means tests with $95 \%$ confidence were calculated. Results: $96.1 \%$ women and 95.6 men were right hand dominant. For cylindrical test, strength increased from 10.3 to $25 \mathrm{~kg}$ average at 17 years of age in women, in men in increased slowly up to 12 years of age reaching $39 \mathrm{~kg}$ at the end of the study. Spherical prehension showed slow, gradual increase reaching 0.45 bar in women and 0.65 bar in men at 17 years of age. Clasp at 17 years of age was 6.9 and $9.5 \mathrm{~kg}$ for women and men respectively. Conclusions: When the right hand is dominant, it is at least $6 \%$ stronger in clasp than the left hand in women, $5 \%$ in fist and clasp in men, with a $95 \%$ confidence between gender between the ages of 7 and 17. These data could be used as reference in patients with pathology that compromises dexterity in urban areas.

(Key words: Force, grasping, manual function, dynamometer, bulb dynamometer, pinch meter, age). Rev Chil Pediatr 2009; 80 (5): 435-443

\section{RESUMEN}

Introducción: Determinar parámetros de normalidad de fuerzas de prensión, permitirá objetivar condiciones clínicas y resultados de tratamientos en pacientes con alteraciones de funcionalidad manual. Objetivo:

Trabajo recibido el 13 de enero de 2009, devuelto para corregir el 05 de mayo de 2009, segunda versión el 02 de julio de 2009, aceptado para publicación el 14 de julio de 2009.

Investigación aprobada y financiada por Instituto de Rehabilitación Infantil Teletón. 
Establecer rangos de normalidad de fuerza en tres tipos de prensión en sujetos de ambos sexos de 7-17 años cumplidos. Materiales y Método: 753 sujetos seleccionados aleatoriamente de colegios de Región Metropolitana, en muestreo bietápico con 2,12\% de error. Se evaluó prensión palmar cilíndrica (dinamómetro), palmar esférica (vigorímetro) y pinza sub-terminolateral (pinzómetro); se probó normalidad estadística para establecer intervalos de confianza de $95 \%$ para las medias. Resultados: $96,1 \%$ de mujeres y $95,6 \%$ de varones declararon mano derecha como dominante. Para prensión cilíndrica, la fuerza creció desde 10,3 hasta $25 \mathrm{~kg}$ promedio a los 17 años en mujeres; en varones, aumentó lentamente hasta los 12 años, alcanzando $39 \mathrm{~kg}$ promedio a igual edad. Prensión esférica presentó crecimiento lento y gradual obteniendo 0,45 bar en mujeres y 0,65 bar en varones de 17 años. En pinza, los valores promedio a la edad máxima, alcanzaron 6,9 y $9,5 \mathrm{~kg}$ para mujeres y hombres respectivamente. Conclusión: la mano derecha cuando es considerada dominante, tiene a lo más, un $6 \%$ de fuerza en pinza mayor que la izquierda en mujeres y de $5 \%$ en fuerza de puño y pinza en hombres; los intervalos con $95 \%$ de confianza para las prensiones estudiadas según género de 7 a 17 años de edad, pueden constituirse en patrones de referencia al comparar las fuerzas de prensión en pacientes con patologías que comprometan función manual con la población normalmente desarrollada de áreas urbanas.

(Palabras clave: Fuerza, prensión, funcionalidad manual, dinamómetro, vigorímetro, pinzómetro, edad).

Rev Chil Pediatr 2009; 80 (5): 435-443

\section{Introducción}

En rehabilitación infantil la intervención está dirigida a estimular el desarrollo y habilidades que permitan un desempeño ocupacional satisfactorio definido como "la habilidad de un individuo para llevar a cabo el conjunto de todas las tareas, rutinas y actividades cotidianas, de acuerdo a sus destrezas, factores personales y las demandas de la actividad y del contexto que lo rodea"1.

La mano, instrumento mecánico de extraordinaria eficiencia, tiene como función principal la prensión y debido a la gran versatilidad de movimiento de la que es capaz, se constituye en el principal órgano para la manipulación física del medio y fuente de información táctil, jugando un rol significativo en el nivel de satisfacción ocupacional.
En relación a los tipos de prensiones usadas en esta investigación, Kapandji ${ }^{2}$ las define de la siguiente forma:

a) Prensiones palmares, tanto cilíndricas como esféricas, en las que intervienen los dedos y palma de la mano. Es una prensión de fuerza para tomar objetos pesados y voluminosos (Figuras 1 y 2 ).

b) Prensiones digitales, referida a la pinza bidigital subtérmino lateral. Involucra pulgar e índice y permite tomar objetos con precisión (Figura 3).

Si consideramos la capacidad funcional de los miembros superiores, uno de los aspectos más importantes involucrados en el desarrollo de la motricidad manual debido a su relación con el desempeño ocupacional, es la fuerza muscular de agarre que determina la eficiencia

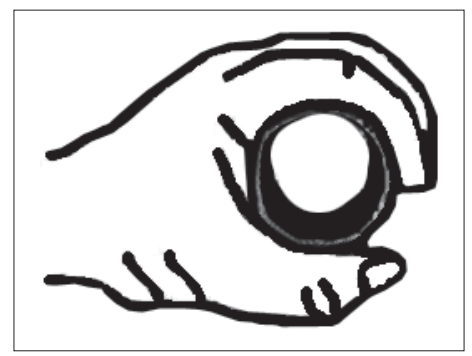

Figura 1. Prensión palmar cilíndrica.

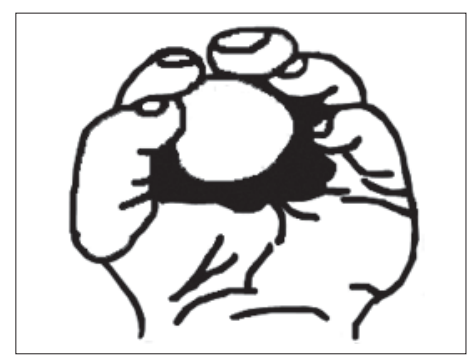

Figura 2. Prensión palmar esférica.

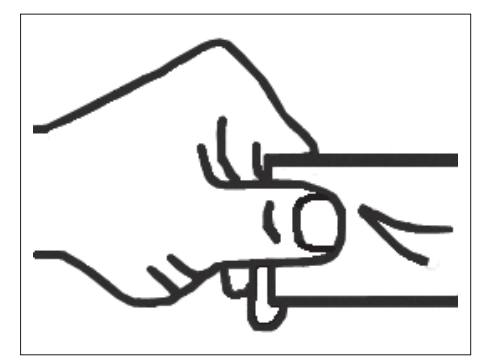

Figura 3. Prensión de pinza. 
en el uso de herramientas y elementos de utilización cotidiana.

Se entiende como fuerza muscular "la capacidad para demostrar el grado de potencia de un músculo cuando al movimiento se le opone resistencia, por ejemplo con objetos o con gravedad" o como "la capacidad de un músculo o grupo muscular para producir tensión y una fuerza resultante en un esfuerzo máximo, de forma dinámica o estática, en relación con las demandas que se le imponen"3.

En Teletón, la evaluación de la fuerza de agarre se realiza en intervención clínica con tres instrumentos para determinar los valores de agarre cilíndrico, de prensión palmar esférica y pinza sub-término lateral, careciendo de patrón de referencia que indique cuanto se desvía la medición de la fuerza de agarre en niños con discapacidades del rango de valores normales correspondientes.

Nuestros resultados no son comparables con la literatura disponible, dado que se trata de universos diferentes (adultos-ancianos versus niños-jóvenes) y adiciona además, fuerzas de agarre esférico y de pinza sub-término lateral. Por otra parte, la mayoría de los autores divide la población en décadas u otros rangos de edades, mientras que el nuestro lo realiza por año cumplido, lo que hace más confiables los valores de la evaluación.

Considerando que la fuerza muscular forma parte de las habilidades de desempeño que tienen propósitos funcionales implícitos ${ }^{4,5} \mathrm{y}$ la importancia de profundizar en el conocimiento de la variación normal de la fuerza prensora en función de la edad y el sexo, se plantea como objetivo general establecer límites de normalidad de fuerza prensora palmar cilíndrica, palmar esférica y pinza bidigital subtérmino lateral en la población de la Región Metropolitana de varones y mujeres entre 7 y 17 años de edad cumplida, estimando que en dichas edades las habilidades motoras básicas y generales asientan las bases de actividades motoras más avanzadas y especificas, permitiendo los progresos de la prensión y de la manipulación, ya que los movimientos se afinan y se diferencian permitiendo que la fuerza y la coordinación aumenten de un modo regular ${ }^{6}$.

\section{Materiales y Método}

\section{Población en estudio}

Sujetos de 7 a 17 años cumplidos, residentes en Región Metropolitana en 2007.

Se excluyeron portadores de alguna patología que pudiera afectar la fuerza muscular de las extremidades superiores o que al momento de la evaluación padecieran molestia, dolor, limitación articular o se encontraran practicando algún deporte o actividad laboral de uso continuo de extremidades superiores.

\section{Muestra}

El marco muestral se obtuvo del registro de establecimientos educacionales de la Región Metropolitana del Ministerio de Educación, incluyendo colegios municipalizados, subvencionados y particulares. El listado incluyó 607 colegios de comunas del área urbana. Los alumnos de $2^{\circ}$ año básico a $4^{\circ}$ año medio, comprendían el universo de las edades a estudiar, totalizando 1118716 niños.

Se realizó un muestreo bietápico. En primer lugar, se sorteó aleatoriamente una comuna de cada zona de la Región Metropolitana: oriente, occidente, sur, norte y centro. En ellas, 86 colegios cumplieron con los requisitos establecidos previamente. La segunda etapa, consistió en la selección aleatoria de colegios. Para calcular el tamaño de muestra, se usó la estimación de proporción poblacional con universo conocido, bajo el supuesto que se muestrearía el $10 \%$ de alumnos en cada edad, con un nivel de confianza de $95 \%$ y un error de $2,12 \%$. Se obtuvo 760 sujetos. Los niños y jóvenes seleccionados, debían contar con el consentimiento informado previamente firmado por el apoderado, quien además completó cuestionario con antecedentes de salud del estudiante.

De los 760 niños examinados, se eliminaron $7(0,9 \%)$ por pertenecer a edades inferiores a 7 años o superiores a 17 años, totalizando 753 evaluados. El 51,1\% correspondió al sexo masculino.

En tabla 1 se observa que el muestreo asignó proporciones similares por edades y sexo; la edad de mayor representatividad fue 15 y la menor 17 años. 
Tabla 1. Distribución de la muestra según edad y sexo

\begin{tabular}{rrrrrrr}
\hline Edad (años) & \multicolumn{2}{c}{ Femenino } & \multicolumn{2}{c}{ Masculino } & \multicolumn{2}{c}{ Total } \\
& $\mathbf{n}$ & \multicolumn{1}{c}{$\%$} & $\mathbf{n}$ & \% & n & \% \\
\hline $7,00-7,99$ & 38 & 10,3 & 36 & 9,4 & 74 & 9,8 \\
$8,00-8,99$ & 35 & 9,5 & 44 & 11,4 & 79 & 10,5 \\
$9,00-9,99$ & 41 & 11,1 & 36 & 9,4 & 77 & 10,2 \\
$10,00-10,99$ & 31 & 8,4 & 29 & 7,5 & 60 & 8,0 \\
$11,00-11,99$ & 33 & 9,0 & 27 & 7,0 & 60 & 8,0 \\
$12,00-12,99$ & 35 & 9,5 & 33 & 8,6 & 68 & 9,0 \\
$13,00-13,99$ & 36 & 9,8 & 33 & 8,6 & 69 & 9,1 \\
$14,00-14,99$ & 26 & 7,1 & 31 & 8,1 & 57 & 7,6 \\
$15,00-15,99$ & 41 & 11,1 & 53 & 13,8 & 94 & 12,5 \\
$16,00-16,99$ & 24 & 6,5 & 36 & 9,4 & 60 & 8,0 \\
$17,00-17,99$ & 28 & 7,6 & 27 & 7,0 & 55 & 7,3 \\
Total & 368 & 100 & 385 & 100 & 753 & 100 \\
\hline
\end{tabular}

\section{Método}

Se consideró la evaluación clínica de la fuerza muscular con el objetivo de medir contracción muscular máxima. Se utilizaron instrumentos de amplia aceptación para evaluar cada una de las prensiones descritas.

a) Dinamómetro de $\mathrm{Jamar}^{7}$, para fuerza de agarre cilíndrico, con registro de fuerza en kilogramos (unidad de masa) (Figura 4).

b) Martín Vigorimeter ${ }^{8}$, para prensión palmar esférica, medido en bar (unidad de presión) (Figura 5).

c) Jamar Hidraulic Pinch Gauge ${ }^{9}$ para evaluar fuerza de pinza; medido en kilogramos (unidad de masa) (Figura 6).

Se determinó la postura sedente como la más adecuada para realizar la evaluación, in- cluyendo columna alineada, hombros aducidos y sin rotación, codo flectado en $90^{\circ}$ a un costado del cuerpo, antebrazo y muñeca en posición neutra (Según Asociación Americana de Terapeutas de mano, 1981) ${ }^{10}$.

Con respecto de la posición del dinamómetro fue determinada según el tamaño de la mano, permitiendo un agarre cómodo y funcional del instrumento con un adecuado cierre de las articulaciones metacarpofalángicas e interfalángicas en la posición de puño, favoreciendo el contacto entre la primera falange de índice y pulgar.

En cuanto al vigorímetro, el tamaño fue seleccionado de tal manera que permitiera un agarre firme y constante del instrumento en la posición de prensión esférica, con antebrazo en supinación.

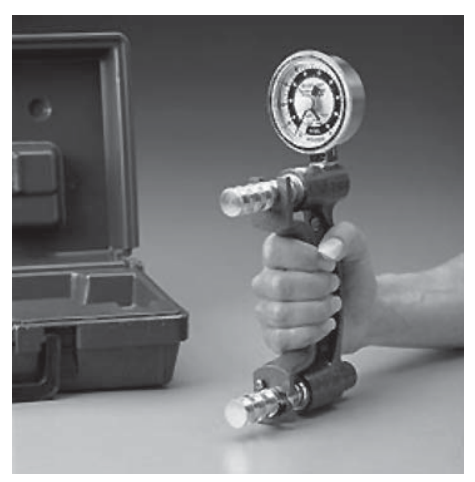

Figura 4. Dinamómetro de Jamar.

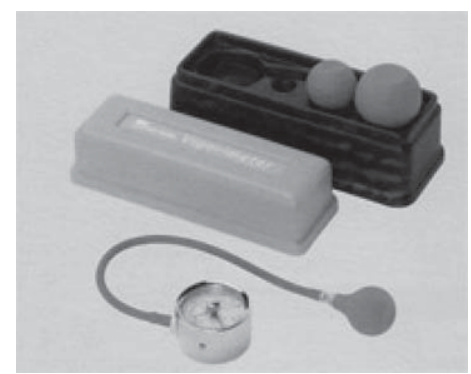

Figura 5. Martin Vigorimeter.

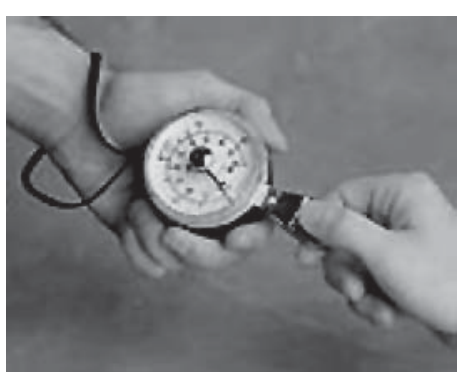

Figura 6. Jamar Hidraulic Pinch Gauge. 
En relación al pinzómetro, fue ubicado frente a la mano evaluada con el fin de permitir una adecuada realización de la pinza lateral entre índice y pulgar en cada uno de los casos.

Primero se evaluó mano derecha y luego izquierda, iniciando las mediciones con dinamómetro, luego vigorímetro y finalizando con pinzómetro. Las variables en estudio fueron medidas en tres oportunidades en cada mano y promediadas para obtener mayor estabilidad en las mediciones. Se consideró un intervalo de 60 segundos como mínimo para favorecer la recuperabilidad fisiológica del músculo y evitar fatiga muscular ${ }^{10}$. Las mediciones fueron realizadas por tres terapeutas ocupacionales entrenadas en el manejo de los instrumentos; se evaluó variabilidad entre ellas mediante análisis de varianza, bajo la hipótesis que en promedio las mediciones de las diferentes variables no diferían entre las terapeutas, hipótesis no rechazada con un valor de alfa $>0,05$.

El análisis estadístico incluyó pruebas de normalidad de Kolmogorov Smirnov, determinación de promedio, desviación estándar e intervalos de confianza de $95 \%$ para la media por sexo y edad. Todas las pruebas con $\mathrm{p}<0,05$.

\section{Resultados}

\section{Análisis de normalidad estadística}

En primera instancia, se estableció que no existían diferencias significativas en la fuerza de agarre en sus tres modalidades para mano derecha e izquierda dentro de cada sexo $(\mathrm{p}>$ $0,05)$ lo que permitió promediar éstos valores de fuerza sin distinguir mano; se obtuvieron distribuciones normales en el 91\% de las varia- bles analizadas; como los tamaños de muestra en cada edad y sexo eran superiores a 25 (tabla 1), se aplicó el teorema central del límite y se optó por describir las diferentes variables de fuerza de agarre con medidas de distribuciones continuas (tabla 2).

\section{Fuerza según mano dominante, dominada $y$ sexo}

Considerando la declaración de uso de mano dominante efectuada por el encuestado, se determinaron las razones mano dominante/mano dominada, según fuese la mano izquierda o derecha. De acuerdo a esta clasificación 29 niñas $(3,85 \%)$ y 33 varones $(4,38 \%)$, expresaron que la mano izquierda predominaba; el resto de la muestra mostró preferencia de uso de mano derecha. En figura 7, se muestra cuando predomina la fuerza de mano derecha sobre la izquierda, es decir, el cociente entre ambas fuerzas, indican que a lo más, la mano derecha tiene un $6 \%$ de fuerza mayor que la izquierda para el caso de la fuerza de pinza en mujeres y de $5 \%$ de puño y pinza en hombres. La mayoría de los promedios de razones de fuerza, son iguales para ambas manos cuando predomina mano izquierda. A título individual, algunos sujetos registraron fuerzas mayores que el promedio.

\section{Intervalos de confianza de 95\% para el promedio}

Como el objetivo del trabajo era obtener valores de normalidad por edad y sexo para las distintas medidas de fuerza de prensión, en tabla 3 se observa los promedios e intervalos de confianza de $95 \%$ en prensión palmar cilíndrica según sexo y edad; en mujeres esta fuerza de

Tabla 2. Medidas de resumen de prensión según mano y sexo

\begin{tabular}{|c|c|c|c|c|c|c|}
\hline Prensión & Mínimo & $\begin{array}{l}\text { Mujeres } \\
\text { Máximo }\end{array}$ & $\mathrm{X} \pm \mathrm{DS}$ & Mínimo & $\begin{array}{l}\text { Hombre } \\
\text { Máximo }\end{array}$ & $\mathrm{X} \pm \mathrm{DS}$ \\
\hline De puño mano derecha & 6,67 & 41,33 & $19,5 \pm 6,7$ & 6,83 & 55,33 & $24,94 \pm 11,72$ \\
\hline De puño mano izquierda & 5,33 & 41,00 & $18,7 \pm 6,46$ & 5,67 & 61,00 & $23,67 \pm 11,02$ \\
\hline Prensión esférica mano derecha & 0,20 & 0,65 & $0,42 \pm 0,08$ & 0,21 & 0,98 & $0,47 \pm 0,14$ \\
\hline Prensión esférica mano izquierda & 0,19 & 0,64 & $0,40 \pm 0,08$ & 0,18 & 0,99 & $0,46 \pm 0,13$ \\
\hline De pinza mano derecha & 2,75 & 9,5 & $5,68 \pm 1,48$ & 2,5 & 15,67 & $6,75 \pm 2,36$ \\
\hline De pinza mano izquierda & 2,42 & 9,58 & $5,37 \pm 1,39$ & 2,5 & 17,00 & $6,46 \pm 2,22$ \\
\hline
\end{tabular}




\section{Razones de fuerza de prensión según sexo}

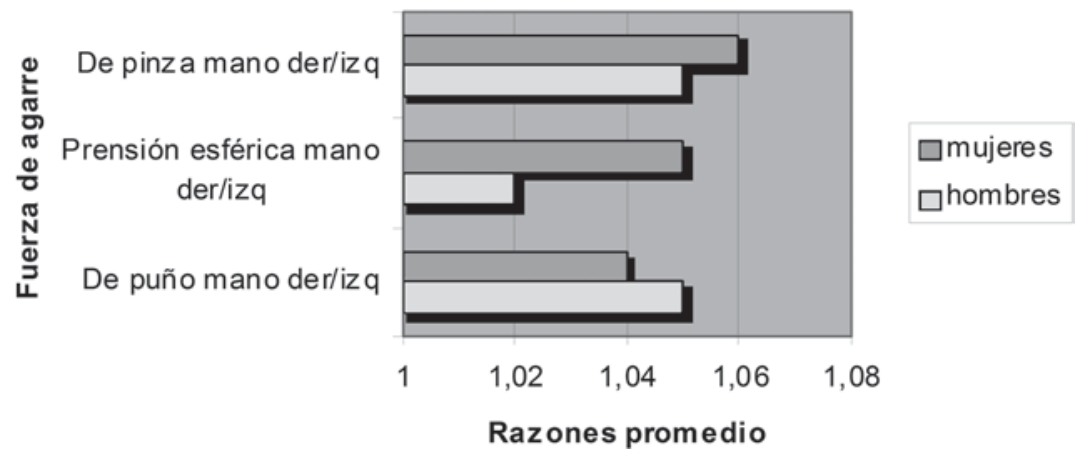

Figura 7. Razones de fuerza de prensión según sexo.

Tabla 3. Fuerza de prensión cilíndrica para ambas manos según sexo y edades

\begin{tabular}{|c|c|c|c|c|c|c|c|c|c|c|}
\hline \multirow[t]{2}{*}{ Edad (años) } & \multirow[t]{2}{*}{$\mathbf{n}$} & \multirow[t]{2}{*}{ Media } & \multirow[t]{2}{*}{$\begin{array}{l}\text { Femenino } \\
\text { DS. }\end{array}$} & \multicolumn{2}{|c|}{$\begin{array}{l}\text { Límites de } \\
\text { confianza } 95 \%\end{array}$} & \multirow[t]{2}{*}{$\mathbf{n}$} & \multirow[t]{2}{*}{ Media } & \multirow[t]{2}{*}{$\begin{array}{l}\text { Masculino } \\
\text { DS. }\end{array}$} & \multicolumn{2}{|c|}{$\begin{array}{l}\text { Límites de } \\
\text { confianza 95\% }\end{array}$} \\
\hline & & & & Lím inf & Lím sup & & & & Lím inf & Lím sup \\
\hline $07,0-07,9$ & 38 & 10,38 & 1,616 & 9,8568 & 10,919 & 36 & 11,25 & 1,6535 & 10,6975 & 11,816 \\
\hline $08,0-08,9$ & 35 & 11,45 & 1,734 & 10,861 & 12,052 & 44 & 12,75 & 2,7295 & 11,9296 & 13,589 \\
\hline $09,0-09,9$ & 41 & 13,73 & 2,942 & 12,811 & 14,668 & 36 & 13,91 & 2,8046 & 12,9631 & 14,861 \\
\hline $10,0-10,9$ & 31 & 16,80 & 3,485 & 15,522 & 18,079 & 29 & 16,45 & 3,6016 & 15,0900 & 17,829 \\
\hline $11,0-11,9$ & 33 & 18,72 & 3,648 & 17,428 & 20,015 & 27 & 18,74 & 3,2634 & 17,4559 & 20,037 \\
\hline $12,0-12,9$ & 35 & 20,94 & 3,242 & 19,833 & 22,061 & 33 & 22,08 & 3,9824 & 20,6687 & 23,492 \\
\hline $13,0-13,9$ & 36 & 23,03 & 4,464 & 21,526 & 24,547 & 33 & 25,74 & 6,4580 & 23,4575 & 28,037 \\
\hline $14,0-14,9$ & 26 & 24,44 & 3,502 & 23,027 & 25,856 & 31 & 32,78 & 5,9499 & 30,6025 & 34,967 \\
\hline $15,0-15,9$ & 41 & 25,99 & 3,936 & 24,753 & 27,238 & 53 & 36,30 & 5,6398 & 34,7505 & 37,859 \\
\hline $16,0-16,9$ & 24 & 25,65 & 4,459 & 23,769 & 27,535 & 36 & 36,81 & 7,5198 & 34,2751 & 39,363 \\
\hline $17,0-17,9$ & 28 & 23,45 & 4,550 & 21,693 & 25,222 & 27 & 39,38 & 5,2374 & 37,1150 & 41,650 \\
\hline Total & 368 & 19,11 & 6,500 & 18,44 & 19,78 & 385 & 24,30 & 11,30 & 23,17 & 25,44 \\
\hline
\end{tabular}

prensión crece constantemente para estabilizarse alrededor de $25 \mathrm{~kg}$. En varones aumenta lentamente hasta los 12 años, luego aumenta su velocidad para mantenerse alrededor de 39,0 $\mathrm{kg}$. en promedio.

La fuerza de prensión esférica, en promedio también aumenta a medida que aumenta la edad, sin embargo, la tendencia en ambos sexos no es lineal; en varones entre los 7 y 11 años, el promedio se mantiene con leves variaciones; a partir de los 12 años, se produce un aumento en la velocidad, para estabilizarse alrededor de
0,48 bar. En mujeres existe mayor dispersión, aunque se visualizan dos grupos de comportamiento: entre 7 y 12 años y desde los 13 hasta 17 años (tabla 4).

En fuerza de pinza, para el sexo femenino, los promedios son inferiores a $5 \mathrm{~kg}$ hasta $10 \mathrm{~s} 11$ años; luego aumentan constantemente hasta los 17 años, para situarse en $6,9 \mathrm{~kg}$. En el sexo masculino, la fuerza de pinza se mantiene bajo $5 \mathrm{~kg}$ hasta los 9 años, aumentando a una mayor velocidad que en mujeres hasta alcanzar $9,5 \mathrm{~kg}$ a los 17 años (tabla 5). 
Tabla 4. Fuerza de prensión esférica para ambas manos según sexo y edades

\begin{tabular}{|c|c|c|c|c|c|c|c|c|c|c|}
\hline \multirow[t]{2}{*}{ Edad (años) } & \multirow[t]{2}{*}{$\mathbf{n}$} & \multirow[t]{2}{*}{ Media } & \multirow[t]{2}{*}{$\begin{array}{l}\text { Femenino } \\
\text { DS. }\end{array}$} & \multicolumn{2}{|c|}{$\begin{array}{l}\text { Límites de } \\
\text { confianza } 95 \%\end{array}$} & \multirow[t]{2}{*}{$\mathbf{n}$} & \multirow[t]{2}{*}{ Media } & \multirow[t]{2}{*}{$\begin{array}{l}\text { Masculino } \\
\text { DS. }\end{array}$} & \multicolumn{2}{|c|}{$\begin{array}{l}\text { Límites de } \\
\text { confianza 95\% }\end{array}$} \\
\hline & & & & Lím inf & Lím sup & & & & Lím inf & Lím sup \\
\hline $07,0-07,9$ & 38 & 0,327 & 0,054 & 0,3093 & 0,3452 & 36 & 0,349 & 0,060 & 0,3289 & 0,3698 \\
\hline $08,0-08,9$ & 35 & 0,364 & 0,069 & 0,3403 & 0,3883 & 44 & 0,379 & 0,070 & 0,3578 & 0,4005 \\
\hline $09,0-09,9$ & 41 & 0,392 & 0,072 & 0,3697 & 0,4153 & 36 & 0,396 & 0,075 & 0,3705 & 0,4215 \\
\hline $10,0-10,9$ & 31 & 0,347 & 0,074 & 0,3196 & 0,3744 & 29 & 0,358 & 0,064 & 0,3338 & 0,3830 \\
\hline $11,0-11,9$ & 33 & 0,384 & 0,060 & 0,3628 & 0,4059 & 27 & 0,354 & 0,065 & 0,3288 & 0,3804 \\
\hline $12,0-12,9$ & 35 & 0,430 & 0,060 & 0,4098 & 0,4511 & 33 & 0,409 & 0,063 & 0,3868 & 0,4322 \\
\hline $13,0-13,9$ & 36 & 0,443 & 0,069 & 0,4200 & 0,4673 & 33 & 0,469 & 0,090 & 0,4379 & 0,5018 \\
\hline $14,0-14,9$ & 26 & 0,446 & 0,052 & 0,4248 & 0,4675 & 31 & 0,535 & 0,097 & 0,4998 & 0,5710 \\
\hline $15,0-15,9$ & 41 & 0,472 & 0,066 & 0,4512 & 0,4932 & 53 & 0,600 & 0,104 & 0,5718 & 0,6292 \\
\hline $16,0-16,9$ & 24 & 0,457 & 0,056 & 0,4334 & 0,4815 & 36 & 0,597 & 0,110 & 0,5600 & 0,6349 \\
\hline $17,0-17,9$ & 28 & 0,458 & 0,067 & 0,4314 & 0,4841 & 27 & 0,650 & 0,104 & 0,6089 & 0,6919 \\
\hline Total & 368 & 0,400 & 0,080 & 0,4000 & 0,4100 & 385 & 0,460 & 0,130 & 23,17 & 25,44 \\
\hline
\end{tabular}

Tabla 5. Fuerza de pinza para ambas manos según sexo y edades

\begin{tabular}{|c|c|c|c|c|c|c|c|c|c|c|}
\hline \multirow[t]{2}{*}{ Edad (años) } & \multirow[t]{2}{*}{$\mathbf{n}$} & \multirow[t]{2}{*}{ Media } & \multirow[t]{2}{*}{$\begin{array}{l}\text { Femenino } \\
\text { DS. }\end{array}$} & \multicolumn{2}{|c|}{$\begin{array}{l}\text { Límites de } \\
\text { confianza } 95 \%\end{array}$} & \multirow[t]{2}{*}{$\mathbf{n}$} & \multirow[t]{2}{*}{ Media } & \multirow[t]{2}{*}{$\begin{array}{l}\text { Masculino } \\
\text { DS. }\end{array}$} & \multicolumn{2}{|c|}{$\begin{array}{l}\text { Límites de } \\
\text { confianza 95\% }\end{array}$} \\
\hline & & & & Lím inf & Lím sup & & & & Lím inf & Lím sup \\
\hline $07,0-07,9$ & 38 & 3,538 & 0,476 & 3,3818 & 3,6950 & 36 & 3,928 & 0,977 & 3,5981 & 4,2598 \\
\hline $08,0-08,9$ & 35 & 4,082 & 0,681 & 3,8479 & 4,3164 & 44 & 4,443 & 0,876 & 4,1772 & 4,7103 \\
\hline $09,0-09,9$ & 41 & 4,506 & 0,623 & 4,3102 & 4,7036 & 36 & 4,816 & 0,723 & 4,5667 & 5,0565 \\
\hline $10,0-10,9$ & 31 & 4,924 & 0,879 & 4,5999 & 5,2449 & 29 & 5,134 & 0,830 & 4,8183 & 5,4501 \\
\hline $11,0-11,9$ & 33 & 5,255 & 0,778 & 4,9789 & 5,5312 & 27 & 5,512 & 0,655 & 5,2529 & 5,7718 \\
\hline $12,0-12,9$ & 35 & 6,246 & 0,859 & 5,9389 & 6,5540 & 33 & 6,347 & 1,090 & 5,9607 & 6,7343 \\
\hline $13,0-13,9$ & 36 & 6,147 & 1,106 & 5,7736 & 6,5223 & 33 & 7,108 & 1,498 & 6,5775 & 7,6401 \\
\hline $14,0-14,9$ & 26 & 6,526 & 0,705 & 6,2412 & 6,8114 & 31 & 8,061 & 1,251 & 7,5571 & 8,4752 \\
\hline $15,0-15,9$ & 41 & 6,798 & 0,810 & 6,5430 & 7,0546 & 53 & 8,741 & 1,353 & 8,3682 & 9,1145 \\
\hline $16,0-16,9$ & 24 & 6,762 & 0,879 & 6,3908 & 7,1335 & 36 & 8,895 & 1,822 & 8,2793 & 9,5124 \\
\hline $17,0-17,9$ & 28 & 6,971 & 0,876 & 6,6314 & 7,3114 & 27 & 9,581 & 1,333 & 9,054 & 10,109 \\
\hline Total & 368 & 5,520 & 1,400 & 5,3800 & 5,6700 & 385 & 6,600 & 2,250 & 6,370 & 6,830 \\
\hline
\end{tabular}

\section{Discusión}

A nivel nacional no se cuenta con parámetros de normalidad de fuerza de agarre en niños y jóvenes con desarrollo psicomotor normal. Esto dificulta precisar la capacidad funcional en sujetos con alteración en la función manual y a posterior evaluar logros en el proceso de rehabilitación y determinar metas, por tanto la determinación de parámetros de fuerza de agarre en niños y jóvenes normales presenta una serie de ventajas para el trabajo en rehabilitación, constituyendo el fundamento de este estudio.

En literatura disponible, los trabajos publicados se refieren principalmente a adultos con evaluaciones de fuerza de agarre cilíndrico, en este trabajo hemos adicionado la medición de fuerzas de agarre esférico y de pinza subtérmino lateral; por otra parte la mayoría de los autores divide la población en rangos de eda$\operatorname{des}^{11,12,13}$, mientras que este estudio se realiza por año cumplido, utilizando instrumentos me- 
cánicos objetivos con réplicas en las mediciones, lo que hace más precisos y confiables los valores determinados.

En adultos, se considera una regla general derivada del estudio de Betchol (1954), citado por Arinci et $\mathrm{al}^{14}$. Esta sugiere que para la fuerza de puño, la mano dominante es aproximadamente un $10 \%$ más fuerte que la no dominante ${ }^{15}$. Nuestros resultados muestran que la mano derecha cuando es considerada dominante, tiene a lo más un 5\% de fuerza mayor que la izquierda en fuerza de puño y pinza de hombres y de $6 \%$ en fuerza de pinza en mujeres y, cuando la mano dominante es la izquierda la fuerza de prensión esférica es superior en $4 \%$ en hombres.

Inicialmente, la fuerza en los niños se desarrolla de forma lenta ya que sus actividades están dirigidas principalmente al área motora gruesa. Hasta los 12 años la fuerza no presenta diferencias en ambos sexos, en adelante el aumento de fuerza se sustenta por el crecimiento fisiológico, manifestado en el crecimiento corporal, aumento de masa muscular, número y velocidad de contracción de fibras del músculo existentes anatómicamente, así como también número y grosor de las miofibrillas desarrolladas ${ }^{16}$.

Esta variabilidad estaría determinada por diferencias de origen estructural y bioquímico originado por cambios hormonales; además de los factores culturales que llevan al varón a un trabajo más dirigido al desarrollo de la fuerza ${ }^{17}$.

Como resumen en los tres tipos de fuerzas evaluadas, a partir de los 14 años, se observaron las mayores diferencias a favor de los varones, determinado principalmente por la diversidad de origen estructural y bioquímica proveniente de los cambios hormonales, además de los factores culturales ya mencionados.

Considerando que la alteración de la fuerza podría llevar a la deformidad articular por desequilibrios entre músculos agonistas y antagonistas, al no abordar esta problemática se produciría una disfunción en las ocupaciones, alterando el desempeño de roles y por ende, el éxito en el cumplimiento de las tareas ocupacionales en los diferentes contextos en que la persona se desempeña; área en que el terapeu- ta ocupacional actúa diseñando un plan de intervención con el fin de mejorar la fuerza y por tanto sus diferentes disfunciones.

Aunque la muestra es sólo representativa de la Región Metropolitana, área esencialmente urbana y haciendo la salvedad del caso que en Regiones podría existir alguna diferencia en la fuerza de prensión por el tipo de trabajo realizado en jóvenes de 15 años o más, hecho por lo demás considerado en los requisitos de inclusión, podríamos inferir que los resultados son representativos de los sujetos chilenos de las edades incluidas de zonas urbanas y servirán de parámetros de normalidad para establecer una guía de trabajo tanto para evaluar como para aplicar alguna intervención; además conocer el comportamiento de la fuerza en relación con el sexo y la edad, nos entrega información que permite regular los niveles de exigencia planteados como objetivos en la planificación terapéutica.

Por último, se debe considerar el desarrollo evolutivo y el rol ocupacional del individuo, estimando la relación de estos ámbitos con la variable fuerza muscular prensora, aplicada a actividades con propósito que den significado a sus roles, entregando un abordaje integral centrado en el individuo, facilitando resultados favorables para un eficiente desempeño en el medio en que se desenvuelve.

\section{Conclusión}

La mano derecha cuando es considerada dominante, tiene a lo más, un $6 \%$ de fuerza en pinza mayor que la izquierda en mujeres y de $5 \%$ en fuerza de puño y pinza en hombres; los intervalos con $95 \%$ de confianza para las prensiones estudiadas según género de 7 a 17 años de edad, pueden constituirse en patrones de referencia al comparar las fuerzas de prensión en pacientes con patologías que comprometan función manual con la población normalmente desarrollada de áreas urbanas; permitiendo optimizar la intervención en el área de la rehabilitación mejorando el planteamiento de objetivos técnicos y la evaluación de logros en terapias, abordajes, procedimientos y tratamientos quirúrgicos utilizados en esta área. 


\section{Referencias}

1.- Trombly CA: Occupational Therapy for physical dysfunction. Williams \& Wilkins.4th edition. pág. 43. 1995. USA.

2.- Kapandji IA: Cuadernos de Fisiología Articular. Miembro Superior. Toray-Masson, 4ª Edición. España.1982.

3.- Crepeau E, Cohn E, Schell B: Terapia Ocupacional. Willard \& Spackman. Editorial médica panamericana. $10^{a}$ edición. España. 2005

4.- Fisher A, Kielhofner G: (1995) skill in Occupational performance. In Kielhofner, G. (1995). A model of human occupation: theory and application. (2nd edition) Baltimore, MD: Williams \& Wilkins.

5.- Marco de trabajo para la Práctica de la Terapia Ocupacional: "dominio y proceso". Asociación americana de Terapia Ocupacional (2002), Revista Americana de Terapia Ocupacional (AJOT) 56, 609-39.

6.- Rigal R: Motricidad humana. Fundamentos y aplicaciones pedagógicas. Editorial Pila Teleña S.A, 1987.

7.- A Patterson Medical Company. Sammons ${ }^{\circledR}$ Preston. Dynamometers [en línea] <http://www.sammons preston.com $>$ [noviembre 2007].

8.- Elmed incorporated. Vigorimeter. http://www.elmed. com/Therapy/vigorimeter.htm [enero 2008].

9.- Jamar Hydraulic Pinch Gauge. < http://www.rehab outlet.com/pinch_gauges.htm $>$ [enero 2008].

10.- Moreira D, Aiza $R$, de Gogoy JR, do Nascimento A: Abordagem sobre prensao palmar utilizando o dinamómetro JAMAR: uma revisao de literatura: Aproach about palmar prehension using dynomemter JAMAR a literature revisión. R Bras Ci e Mov Brasilia 2003; 11 (2): 95-9.

11.- Käthe J, Romero $C$ : Evaluación de la fuerza de puño en sujetos adultos sanos mayores de 20 años de la Región Metropolitana. Tesis (Licenciado en Kinesiología). Santiago, Chile. Universidad de Chile, Facultad de Medicina, 2005. $52 \mathrm{~h}$.

12.- Gómez LS, Navarrete CE, Sepúlveda CI, Lobos AE: Datos normativos de la fuerza de agarre en sujetos en edad laboral en el Área Metropolitana. Revista Chilena de Rehabilitación de la Mano 1994; (38): 12-20.

13.- Anales de Pediatría Vol. 70 Núm. 04 Dinamometría en niños y jóvenes de entre 6 y 18 años: valores de referencia, asociación con tamaño y composición corporal.

14.- Arinci N, Ceceli E, Bakici P, Rana H, Rezan Z: Grip strength: Effect of hand dominance. Singapore Med J 2000; 43: 234-7.

15.- Petersen P, Petrick P, Connor H, Conklin D: Grip strength and hand dominance: challenging the $10 \%$ rule. AJOT 198943 (7): 444-7.

16.- Ojeda Sahagún JL, Icardo De La Escalera JM: Neuroanatomía humana aspectos funcionales y clínicos. Cap 16 pg 199-207.

17.- Devaud N, Spotorno A: Medicina evolutiva del desarrollo puberal. Rev Chil Pediatr 2004; 75 (4): 373 8.

Anexo 1

\section{Autorización de padres/Consentimiento Informado}

\section{AUTORIZACIÓN DE PADRES CONSENTIMIENTO INFORMADO}

El Instituto de Rehabilitación Infantil, Teletón Santiago, en su permanente interés por cumplir con el compromiso de rehabilitar a niños con discapacidad, se encuentra desarrollando una investigación que consiste en la validación de una pauta de evaluación funcional de mano para niños entre 7 y 17 años de edad.

Este estudio científico se encuentra a cargo de 3 Terapeutas Ocupacionales, profesionales de Teletón Santiago, con larga trayectoria en el área de rehabilitación, María Verónica Lagos, Pamela Escalona y Jeannette Naranjo.

Una de las áreas a evaluar en esta investigación es la fuerza de mano, por lo que necesitamos contar con una referencia de fuerza en niños normales, que tengan edad dentro del rango mencionado.

Esta medición, es un procedimiento sencillo, de rápida administración, donde cada niño es evaluado con 3 instrumentos que no implican daño, incomodidad, riesgo ni dolor alguno para su hijo(a).

La participación en este estudio no implica ningún tipo de cobro ni pago por los procedimientos realizados, la información que se obtendrá es parte de este proyecto de investigación y podrá ser utilizada en publicaciones o presentaciones científicas a estudiantes y profesionales de la salud y, en ningún caso, se utilizará el nombre o imagen alguna que permita identificar a los participantes.

Su colaboración significa un gran aporte que beneficia a los niños discapacitados que se atienden en Teletón

Yo,................................................................ RUT...................................... he leído la información descrita y, mis preguntas acerca del estudio han sido respondidas satisfactoriamente. Al firmar esta copia, indico que tengo un entendimiento claro del proyecto y deseo participar en él.

FIRMA APODERADO:

FECHA

Colegio 